\title{
Medical Image of the Month: Double Aortic Arch
}

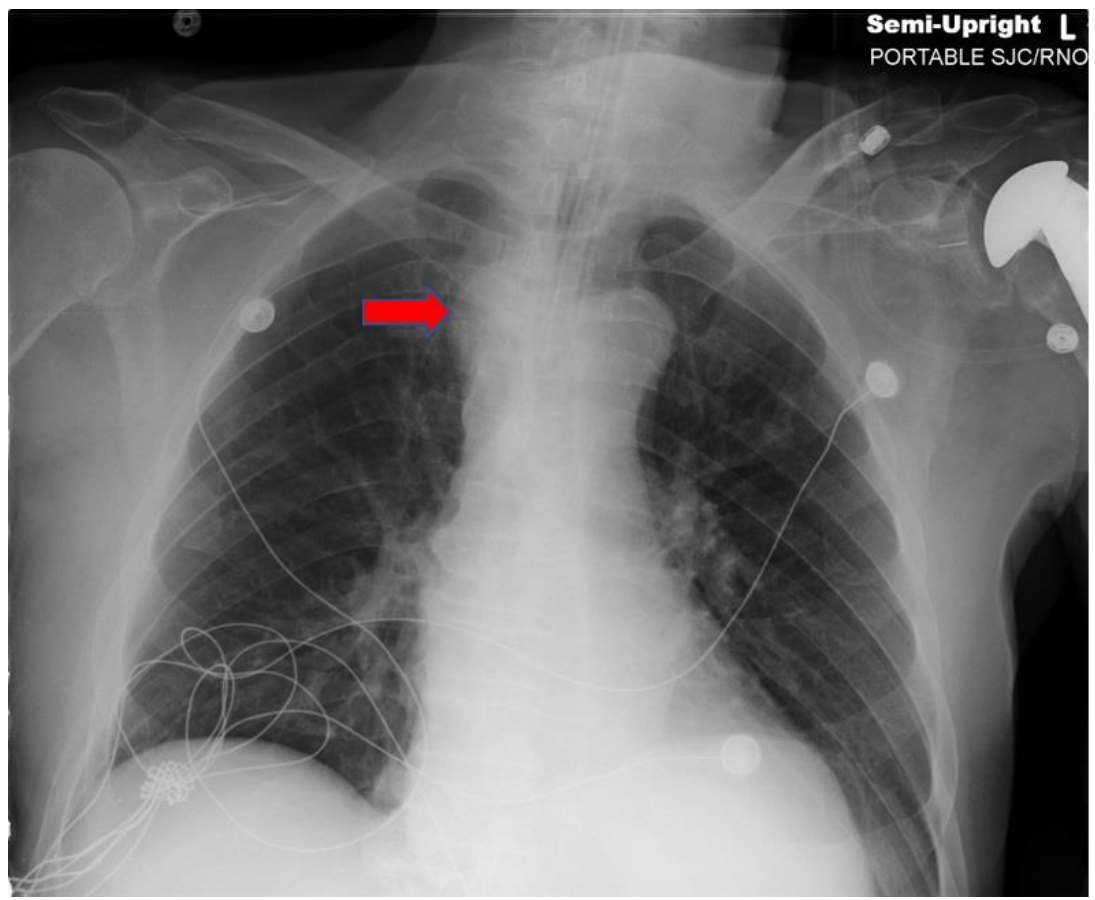

Figure 1. A chest radiograph performed after endotracheal intubation of the patient demonstrated prominence of the upper mediastinum in the region of the right paratracheal strip (red arrow).

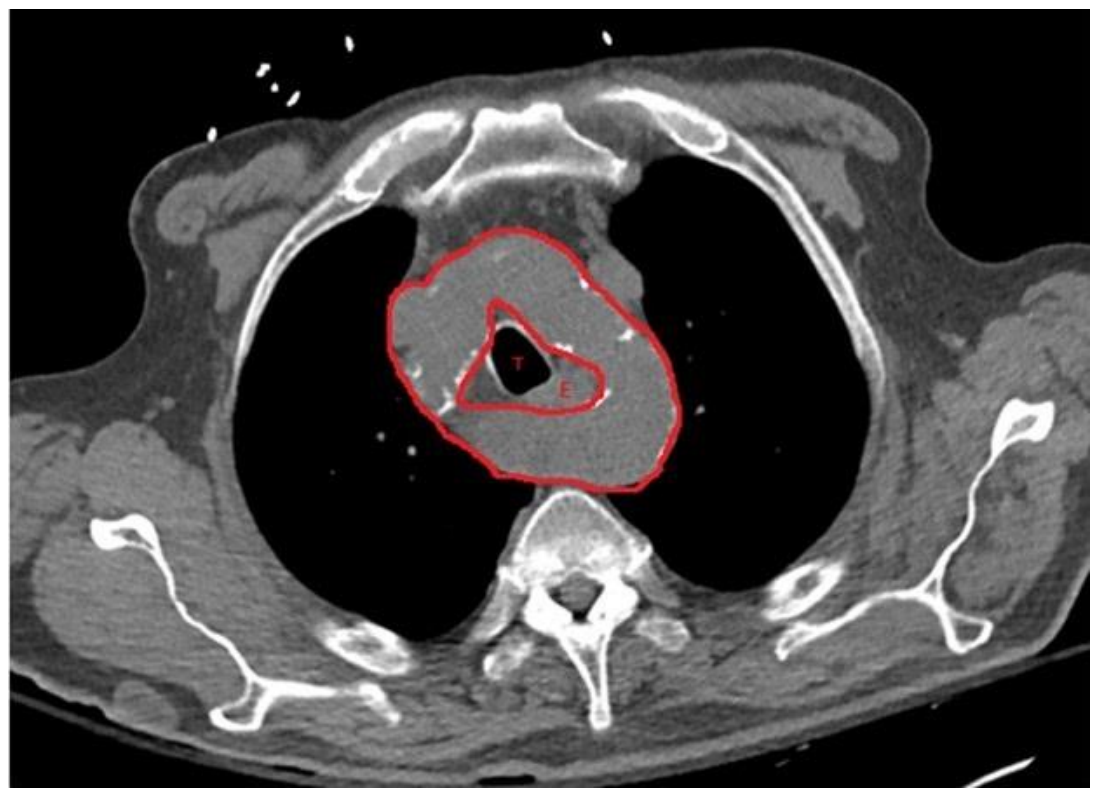

Figure 2. A non-contrasted CT of the chest demonstrates a complete vascular ring secondary to the patient's double aortic arch (outlined in red) surrounding the trachea (T) and esophagus (E). 
Case Presentation: A 78-year-old gentleman presented to the hospital via EMS with altered mental status. An urgent CT of the head performed in the emergency room demonstrated a large, right intraparenchymal hemorrhage with intraventricular extension into the right lateral ventricle. His Glascow Coma Scale score was 6 , and he was intubated for airway protection. A chest radiograph performed to verify placement of the endotracheal tube demonstrated prominence of the upper mediastinum in the region of the right paratracheal strip (Figure 1). A CT of the chest (Figure 2)

demonstrated a double aortic arch corresponding to the upper mediastinal abnormality noted on the chest radiograph. In speaking with the patient's family after acquiring the CT of the chest, they stated that the patient had long-term issues with dysphagia specifically choking with solid foods. Unfortunately, the patient passed away from complications of his large intraparenchymal hemorrhage.

A double aortic arch results from persistence of both the right and left embryonic arches with each arch giving rise to the ipsilateral, separate carotid and subclavian arteries (1). A double aortic arch is the most common cause of a symptomatic vascular ring with the trachea and esophagus being compressed by the two arches (1). Symptoms usually arise in childhood with good outcomes with surgical repair of this abnormality in pediatric populations. A few case series exist describing repair of this anomaly in adult populations (2).

Kelly Wickstrom DO, Steven P. Sears DO, and Laura Meinke MD Division of Pulmonary, Critical Care, Allergy and Sleep Medicine University of Arizona College of Medicine Tucson, AZ USA

\section{References}

1. Hanneman $\mathrm{K}$, Newman $\mathrm{B}, \mathrm{Chan} \mathrm{F}$. Congenital variants and anomalies of the aortic arch. Radiographics. 2017 Jan-Feb;37(1):32-51. [CrossRef] [PubMed]

2. Noguchi K, Hori D, Nomura Y, Tanaka H. Double aortic arch in an adult. Interact Cardiovasc Thorac Surg. 2012 Jun;14(6):900-2. [CrossRef] [PubMed] 